\title{
Bioactive Factors of Colostrum and Human Milk Exhibits a Day-Night Variation
}

\author{
Nubia Andrade Silva, \\ Adenilda Cristina Honorio-Franca, Fernanda Regina Giachini, \\ Luana Mores, Estefani Goncalves de Souza and Eduardo Luzia Franca \\ Department of Biomedicine, Institute of Biological and Health Science, \\ Araguaia University Center, Federal University of Mato Grosso, \\ Rodovia Br 070, Km 5 s/nº, Barra do Garcas-MT, 78600-000, Brazil
}

Received 2013-05-16, Revised 2013-06-24; Accepted 2013-06-25

\begin{abstract}
The aim of this study was to determine the chronobiological variations of cytokines (IFN- $\gamma$, TGF- $\beta$ ) and hormones (melatonin and cortisol). The sample was collected from 42 mothers at three stages of maturity-day 3 (colostrum), day 10 (transitional milk) and day 30 (mature milk) postpartum, at two times of day: diurnal $(12: 00 \mathrm{~h})$ and nocturnal $(24: 00 \mathrm{~h})$, resulting a total of 252 samples. Melatonin concentration was increased in the nocturnal period from all milk maturation stages. This hormone concentration was lower in the mature milk. Cortisol concentration was higher in the mature milk during the diurnal period and it was lower in the nocturnal period when compared to colostrum and transitional milk. IFN- $\gamma$ concentration did not vary between all periods. The transitional milk displayed higher concentrations of this cytokine in the nocturnal period whereas the concentration of IFN- $\gamma$ decreased in the nocturnal period from the mature milk. When comparing the types of milk, it was observed lower concentrations of IFN- $\gamma$ in mature milk in both periods. No significant variation in TGF- $\beta$ concentration was detected between types of milk or at either time of day. These data support the hypothesis that there is a fluctuation in the production of hormone and cytokines and this leads to a need that adequate time of breastfeeding being crucial to ensure passive transfer of immunity, as well as for establishment of synchronization of newborn.
\end{abstract}

Keywords: Chronobiology, Breast Milk, Cytokines, Hormones

\section{INTRODUCTION}

Breastfeeding has been proven to protect infants against a wide range of infectious and noninfectious diseases. Therefore, efforts have been directed to identify the various immunoactive substances in human breast milk that account for its protective effects (Hanson and Silfverdal, 2009; Honorio-Franca et al., 2013).

The chemical composition of the human milk is modified according to pregnancy maturation (prepartum and postpartum) and the time of day (night versus day time), allowing adaptations to the infant's nutritional needs. The composition is also modified during feeding and according to any diseases experienced by the mother (Franca et al., 2010; 2013; Morceli et al., 2011; 2013). Mother's milk can be classified as early lactation (260 days of gestation), colostrum (1 to 7 days postpartum), transitional milk ( 7 to 15 days postpartum) and mature milk (15 days postpartum) (Franca et al., 2010; 2013).

Human milk was first used clinically as a vehicle for passive immunity transfer, but its immune components are now known to be highly immunoreactive, Corresponding Author: Eduardo Luzia Franca, Department of Biomedicine, Institute of Biological and Health Science, Araguaia University Center, Federal University of Mato Grosso, Rodovia Br 070, Km $5 \mathrm{~s} / \mathrm{n}^{\circ}$, Barra do Garças-MT, 78600-000, Brazil 
exhibiting time-dependent alterations (Franca et al., 2010). Fluctuations in the milk content increases progressively up to 3 months postpartum, producing a rhythm that is assumed to stabilize the newborn's circadian system while other controlling mechanisms such as the adults rest-activity cycle are not fully developed (Franca et al., 2012; Honorio-Franca et al., 2013). Breastfeeding provides the bond between mother and child and helps in the neonate synchronization, regulating sleep, food, among others parameters (Chirico et al., 2008; Castellote et al., 2011).

The literature has reported the modulation of immune function as well as the synthesis of regulatory products through the biological rhythms, which include several factors such as hormones and cytokines (Arjona and Sarkar, 2008). The circadian rhythm plays an important role in daily control of hormones such as melatonin and cortisol, which are synchronized internally (Lack and Wright, 2007). These hormones act on function and production of several cytokines, among them interferon gama (IFN- $\gamma$ ) and Transforming Growth Factor beta (TGF- $\beta$ ) (Scheff et al., 2010). However few studies have investigated the chronobiological variable of hormones and cytokines present in the human milk.

It is know that at birth, newborns are essentially noncircadian, probably because the input/output pathways are undeveloped. As an example, immature temporal control is revealed by the lack of consolidated sleep, rhythms in hormonal secretion and body temperature variations (Rivkees, 2003). At this stage, the hormones and cytokines present in milk are important for protection and indicate time of day to breastfed infants.

The constitutional differences of postpartum milk suggest that this secretion undergoes through chronobiological variation. Determining the presence of hormones and cytokines during breastfeeding is important to allow adequate passive transfer of immunity and intake of immunological components as well as synchronization of newborn.

In the present study we evaluated the effects of the time collection (diurnal/nocturnal) on the different types of milk, with regard to the hormones melatonin and cortisol, as well as the cytokines IFN- $\gamma$ and TGF- $\beta$.

\section{MATERIALS AND METHODS}

\subsection{Subjects}

After an informed consent form had been signed by the volunteers, about $15 \mathrm{~mL}$ of colostrum was collected from clinically healthy women, 18-35 years of age (Honorio-Franca et al., 2013), at the Health System Program of Barra of Garcas, Mato Grosso, Brazil. All the mothers had given birth to healthy term babies. The collections were established according to protocol by (Franca et al., 2010). Two breast milk samples were collected from each mother at day 3 postpartum (colostrum), day 10 postpartum (transitional milk) and day 30 postpartum (mature milk), resulting in a total of 252 samples. All procedures were submitted for ethical evaluation and obtained Institutional approval. The variables controlled during pregnancy were smoking status (yes/no), arterial hypertension (yes/no) and glycemia (yes/no).

\subsection{Obtaining Supernatant from Human Milk}

Milk supernatant samples of different puerperae, collected in two periods of time and three stage of maturation were obtained by centrifugation (10 $\mathrm{min}$, $\left.160 \mathrm{xg}, 4^{\circ} \mathrm{C}\right)$. The upper fat layer was discharged and the aqueous supernatant stored at $-80^{\circ} \mathrm{C}$ for later hormone and cytokines analyses.

\subsection{Melatonin and Cortisol Hormone Dosage by an ELISA (Enzyme Linked Immuno Sorbent Assay) Method}

Melatonin was extracted by affinity chromatoraphy, concentrated in speed-vacuum. Melatonin and cortisol concentration were determined using an specific ELISA kit (Immuno-Biological Laboratories, Hamburg-Germany). The reaction rates were measured by absorbance in a spectrophotometer for each plate with a $405 \mathrm{~nm}$ filter. The results were calculated according to the respective standard curve and the results are shown as $\mathrm{pg} / \mathrm{ml}$ (melatonin) and $\mu \mathrm{g} / \mathrm{dL}$ (cortisol).

\subsection{Cytokine Dosage by ELISA (Enzyme Linked Immunosorbent Assay)}

IFN- $\gamma$ concentrations in the colostrum and milk supernatants were determined by an ELISA kit from BioLegend ${ }^{\circledR}$ Ledend Max $^{\mathrm{TM}}$ (San Diego, USA) and TGF- $\beta$ concentrations were analyzed using an ELISA kit from Enzo ${ }^{\circledR}$ Life Sciences (United Kingdom). The reaction rates were measured by absorbance in a spectrophotometer with a $450 \mathrm{~nm}$ filter. The results were calculated using the standard curve and shown in $\mathrm{pg} / \mathrm{dL}$. 


\subsection{Statistical Analysis}

Two-way Analysis of Variance (two-way ANOVA) was used to evaluate the hormone and cytokines concentration, considering the post-partum stage as one factor and time of day as the other. Statistical significance was considered when $\mathrm{p}<0.05$.

\section{RESULTS}

\subsection{Subject Characteristics}

During this study 42 mothers were evaluated with the average of $25.7 \pm 6.1$ years-old. The women gave birth at $38.9 \pm 1.4$ week's gestation. Newborn weight was $3280 \pm 400 \mathrm{~g}$.

\subsection{Melatonin Concentration in Colostrum and Human Milk}

Melatonin concentration in all the milk maturation stages increased in the nocturnal period compared to the diurnal period. In the samples obtained in the diurnal period, melatonin concentration was lower in mature milk compared to both colostrum and transitional milk, which had similar amounts of this hormone. The transitional milk present increase in melatonin concentration in the nocturnal period (Fig. 1).

\subsection{Cortisol Concentration in Colostrum and Human Milk}

Cortisol concentration in the colostrum and transitional milk did not differ among the periods, whereas the mature milk displayed higher concentrations of this hormone in the diurnal period. Cortisol concentration in mature milk was higher during the diurnal period, whereas in the nocturnal period cortisol concentration was lower, when compared to colostrum and transitional milk (Fig. 2).

\subsection{IFN- $\gamma$ Cytokine Concentration}

IFN- $\gamma$ concentration in colostrum did not vary between the periods. The transitional milk displayed higher concentrations of this cytokine in the nocturnal period whereas in the mature milk, smaller concentration of IFN- $\gamma$ were obtained in the nocturnal period. When comparing the types of milks, lower concentrations of IFN- $\gamma$ were observed in mature milk in both periods (Table 1).

\subsection{TGF- $\beta$ Cytokine Concentration}

No significant variation was detected in the TGF- $\beta$ concentration between the different types of milk or at either time of day (Table 1).

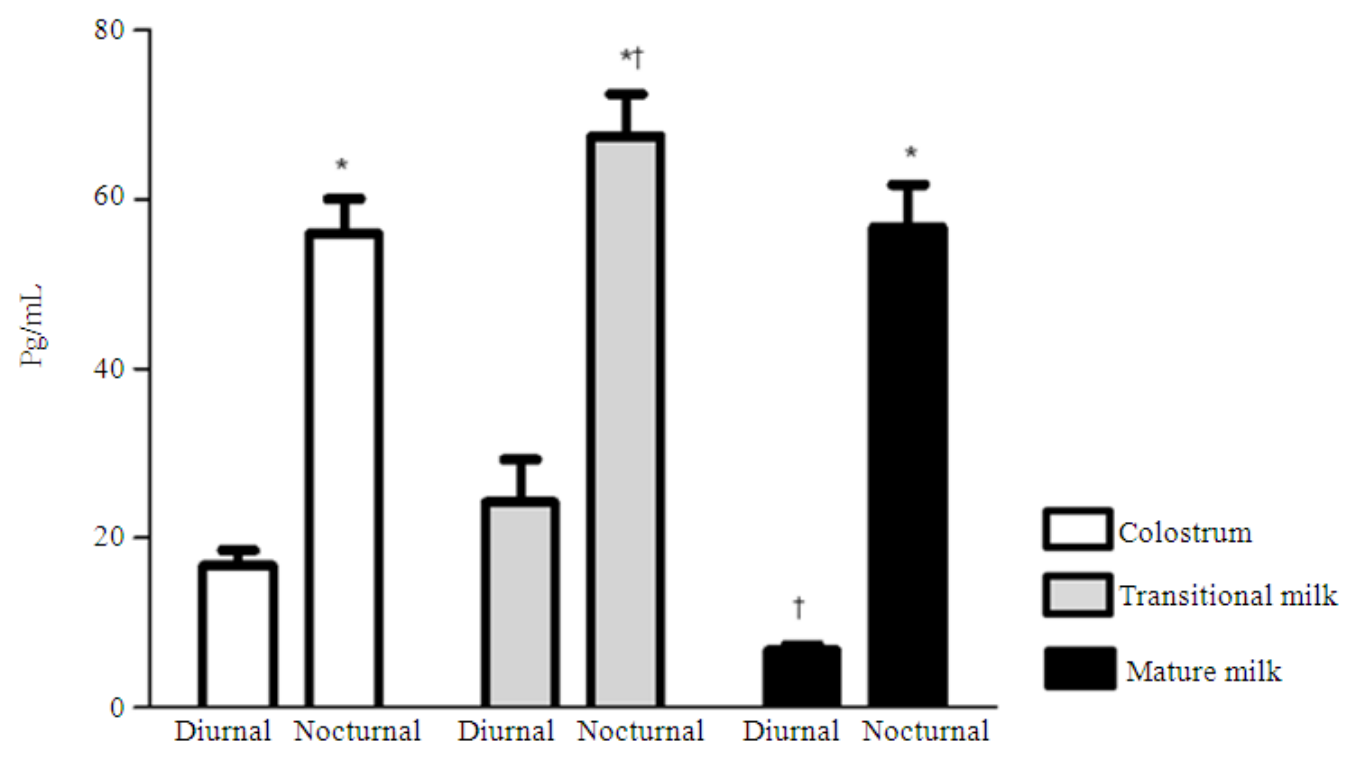

Fig. 1. Melatonin concentration in colostrum, transitional Milk and mature Milk collected in the diurnal and nocturnal periods. Results are expressed as the mean and SD. F $(1,24)=185.4295 ; p=0.0001$ (time of Day) $F(2,24)=6.5410 ; p=0.0056$ (kind of milk). *comparing diurnal and nocturnal phases for the same stage of milk maturation. †comparing colostrum to milk in a same period 


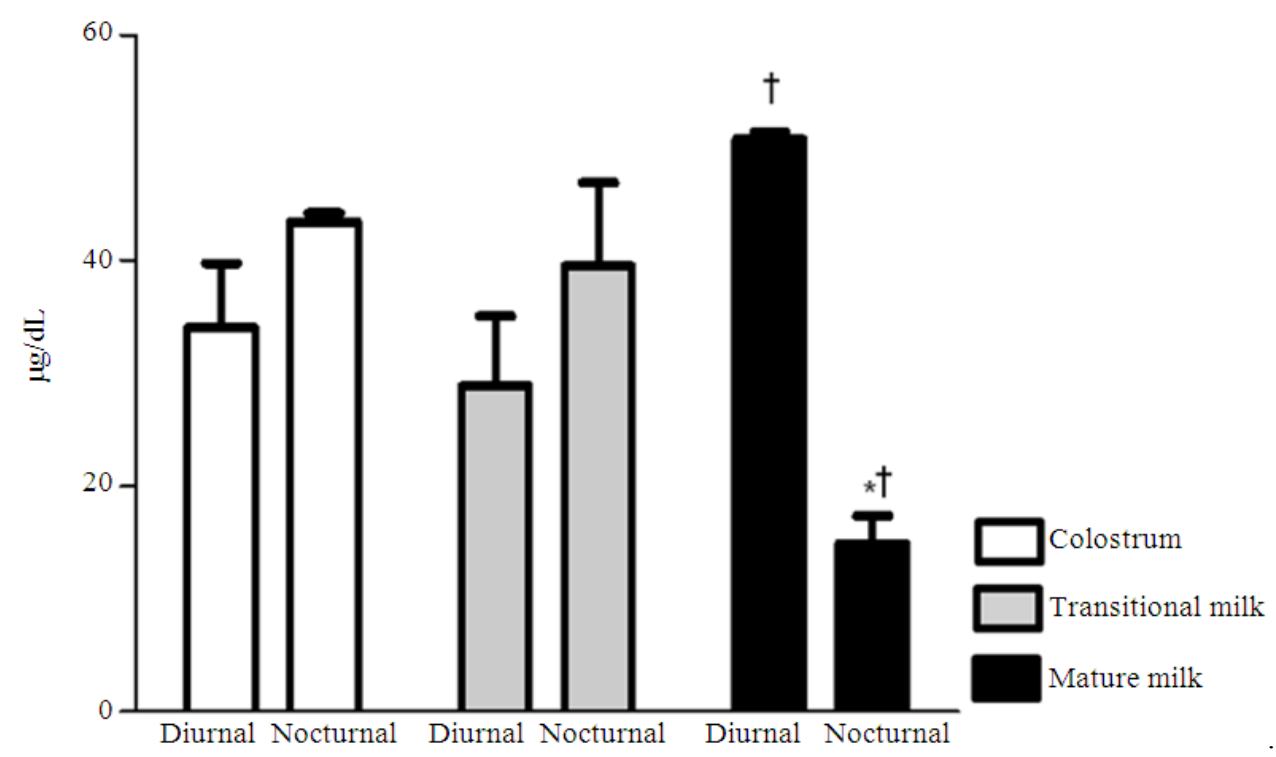

Fig. 2. Cortisol concentration in colostrum, transitional Milk and mature Milk collected in the diurnal and nocturnal periods. Results are expressed as the mean and SD. F $(1,24)=15.7331 ; p=0.0001$ (time of Day) $F(2,24)=7.0108 ; p=0.0006$ (kinf of milk). *comparing diurnal and nocturnal phases for the same stage of milk maturation. †comparing colostrum to milk in a same period

Table 1. IFN- $\gamma$ and TGF- $\beta$ concentrations in colostrum, transitional and mature milk collected in the morning and at night

\begin{tabular}{|c|c|c|c|c|c|}
\hline Cytokine & Phases & Colostrum & Transitional milk & Mature milk & Statistical \\
\hline \multirow[t]{2}{*}{$\overline{\mathrm{IFN}-} \gamma(\mathrm{pg} / \mathrm{dL})$} & Diurnal & $66.3 \pm 14.1$ & $40 \pm 3.7 \dagger$ & $39.9 \pm 14.8 \dagger$ & $\begin{array}{l}F(1,24)=10.0071 \\
P=0.0001\end{array}$ \\
\hline & Nocturnal & $73.3 \pm 16.4$ & $51.2 \pm 22.8^{*}$ & $26.0 \pm 15.6^{* \dagger}$ & $\begin{array}{l}\text { (time of day) } \\
\mathrm{F}(2,36)=14.9432 ; \\
\mathrm{P}=0.0058 \text { (kind of milk) }\end{array}$ \\
\hline \multirow[t]{2}{*}{ TGF- $\beta$ (pg/dL) } & Diurnal & $22.8 \pm 2.0$ & $22.8 \pm 7.0$ & $26.4 \pm 6.1$ & $\begin{array}{l}F(1,24)=0.2063 \\
p=0.6578 \text { (time of day) }\end{array}$ \\
\hline & Nocturnal & $24.2 \pm 4.2$ & $21.3 \pm 1.0$ & $28.3 \pm 9.8$ & $\begin{array}{l}\mathrm{F}(2,24)=2.1500 \\
\mathrm{P}=0.1368 \text { (kind of milk) }\end{array}$ \\
\hline
\end{tabular}

Results were expressed as mean and SD. *Significant differences between periods (day and night), considering the same type of milk. $\dagger$ Significant differences between types of milk, considering the same phase (day or night).

\section{DISCUSSION}

The present study shows that the concentrations of hormone and cytokines of human colostrum and breast milk change as a function of time of day, as well as according to the milk maturation. Human milk has been thoroughly studied in recent years. For nursing babies, it represents an important source of cytokines and hormones (Garofalo, 2010).

The components of human colostrum and breast milk are important for children and may vary over time in some of its components, as well as during the course of feeding (Franca et al., 2010). In the present study, we confirm the fluctuation of melatonin levels in all the milk maturation stages. Melatonin concentrations in milk were higher at night than during the day, confirming previous studies that compared diurnal and nocturnal melatonin concentration in mother's milk (Honorio-Franca et al., 2013).

Newborns have lower melatonin levels, which increase progressively up to the $3 \mathrm{rd}$ month of age, when a characteristic circadian rhythm is detectable (Attanasio et al., 1986). Accordingly, some authors propose that a primary role of milk melatonin is to stabilize the newborn circadian rhythm (Hara et al., 2013; Honorio-Franca et al., 2013) while other biological phenomena such as rest-activity or sleep-wake cycles need to be firmly established. 
Melatonin contained in the human milk is likely derived from blood and plays an important role in newborn synchronization with the mother's rhythm (Franca et al., 2010). Milk melatonin may provide information on day/night cycles to the child until this rhythm is established. The melatonin contained in maternal milk can contribute to protecting the gastrointestinal mucosa of newborns and the daily fluctuations of this exogenous hormone over a $24 \mathrm{~h}$ period, due to breastfeeding, might contribute to the synchronization between the child's rhythms and those of its mother (Franca et al., 2010; 2012; Honorio-Franca et al., 2013).

Neurohormonal control is significantly responsible for driving the immunological effects (Markus and Ferreira, 2011). There are reports in the literature showing that the hormone cortisol is present in the colostrum (Fagundes et al., 2012) and that the endogenous release of this hormone can directly modulate immune function and play a central role in the immune response (Kohut et al., 2005). In vitro assays revealed that at low concentrations glucocorticoids have an effect on the immunostimulatory activity whereas at high concentrations they have an immunosuppressive effect (Lim et al., 2007; Fagundes et al., 2012).

Studies have shown that the mammary gland is responsible for the secretion of hormones that are important for the development and maturation of the gastrointestinal tract and immune system of newborns (Bernt and Walker, 2009). These hormones temporarily regulate the activity of some endocrine glands until the hormonal system of the newborn is fully developed (Sauter et al., 2004). Therefore, the hormones secreted in the milk are considered necessary for the health and growth of newborns and are important modulators of immune functions.

Several studies has reported that cortisol hormone, present in the milk, increase during the day and decrease in nocturnal period in mother's milk (Haus and Smolensky, 2006; Haus, 2007). In the present study, the concentration of cortisol in mature milk was higher during the diurnal period and lower in nocturnal period only in the mature milk. No variation was observed in colostrum and transitional milk, showing that the secreted cortisol acquire synchronization in a later stage of milk maturation. The passive transfer of hormone to the newborn may auxiliary in the mechanisms of immune protection, as well as the maturation of the gastrointestinal tract of infants (Xu et al., 2011). This pioneering study compared the hormonal variation in human milk following the same mothers in different stage of maturation, allowing better comparisons between the hormone secretion profile through the different stages of milk maturation.

The hormones received passively through breastfeeding also provides other benefits such as immunomodulation of the immune system to regulate the production or to stimulate increased expression of several cytokines (Carrillo-Vico et al., 2005).

In this study, IFN- $\gamma$ concentration had higher concentrations in the nocturnal period in transitional milk whereas in mature milk this cytokines decreased. In general, there were lower concentrations of in the mature milk in both periods. In the literature, the data regarding IFN- $\gamma$ is controversial. Some authors have related that this cytokine did not show variation in the concentration between the stage of maturation (Prokesova et al., 2006), while others have reported that these cytokines are found at different concentrations in stages lactation (Groen and Beckstead, 2011).

Despite the decreased of IFN- $\gamma$ in mature milk, it should be considered that the rate of cytokine is related to the total amount of milk that the infant consume, rather than the concentration of them in the milk. Therefore, adequate duration of breastfeeding is crucial to ensure for passive transfer of immunity components to newborn.

Colostrum contains several cytokines and growth factors, some of which exert inhibitory or stimulants effects on immune responses (Garofalo, 2010). The ability of human milk provide passive immunity and actively modulate the development of the immune system and systemic mucosa of the newborn, is closely linked to microbicidal, anti-inflammatory and immunomodulatory properties of cytokine (Garofalo, 2010).

Some cytokines have been associated with regulation of development and function of the mammary gland (Watson et al., 2011) while others may be linked with production of defense, or/and other cytokines by the mammary glands. There are various signals associated cytokines in human milk (Garofalo, 2010). The TGF- $\beta$ concentrations have been related in the human milk. Studies showed that this cytokine decreased during the stage of maturation, with the highest concentrations in colostrum (Castellote et al., 2011).

Here, we have demonstrated that TGF- $\beta$ is present in the human milk and no variation of this cytokines was detected among the different stages of lactation or at either time of day. These results are in agreement with the literature, 
where it has been reported that TGF- $\beta$ concentrations remains unaltered on different stages of lactation (Prokesova et al., 2006; Groen and Beckstead, 2011).

The present study suggests the existence of a circadian rhythms in soluble components of human milk. This chronobiological variation also changes during the course of the first month postpartum. Such variation may represent an additional breastfeeding mechanism to improve newborn adaptation to environmental changes.

\section{CONCLUSION}

These data support the hypothesis that there is a fluctuation in the production of hormone and cytokines at different stages of milk maturation and this fact leads to a necessity to adequate the time of breastfeeding, being crucial to ensure passive transfer of immunity and might represent an endogenous force promoting the establishment of biological rhythmicity in the human.

\section{ACKNOWLEDGMENTS}

This researches received grants from Fundacão de Amparo à Pesquisa de Mato Grosso (FAPEMAT No 299032/2010) and Conselho Nacional de Pesquisa (CNPq N 475826/2010-8; N 475739/2011-6).

\section{REFERENCES}

Arjona, A. and D.K., Sarkar, 2008. Are Circadian rhythms the code of hypothalamic-immune communication? insights from natural killer cells. Neurochemical Res., 33: 708-718.

Attanasio, A., K. Rager and D. Gupta, 1986. Ontogeny of circadian rhythmicity for melatonin, serotonin and Nacetylserotonin in humans. J. Pineal Res., 3: 251-256. DOI: 10.1111/j.1600-079X.1986.tb00747.x

Bernt, K.M. and W.A. Walker, 2009. Human milk as a carrier of biochemical messages. Acta Paediatr., 88: 27-41. DOI: 10.1111/j.1651-2227.1999.tb01298.x

Carrillo-Vico, A., J.M. Guerrero, P.J. Lardone and R.J. Reiter, 2005. A review of the multiple actions of melatonin on the immune system. Endocrine, 27: 189-200. DOI: 10.1385/ENDO:27:2:189

Castellote, C., R. Casillas, C. Ramirez-Santana, F.J. PerezCano and M. Castell et al., 2011. Premature Delivery influences the immunological composition of colostrum and transitional and mature human milk. J. Nutr., 4: 1181-1187. DOI: 10.3945/jn.110.133652
Chirico, G., R., Marzollo, S., Cortinovis, C., Fonte and A. Gasparoni, 2008. Antiinfective properties of human milk. J. Nutr., 138: 1801S-1806S. PMID: 18716190

Fagundes, D.L.G., E.L. Franca, C.C.P. Hara and A.C. Honorio-Franca, 2012. Immunomodulatory Effects of poly (ethylene glycol) microspheres adsorbed with cortisol on activity of colostrum phagocytes. Int. J. Pharmacol., 8: 510-518. DOI: 10.3923/ijp.2012.510.518

Franca, E.L., C.C.P. Hara, D.L.G. Fagundes, N.A.P. Lima, S.H.B. Ratto, A.C. Honorio-Franca, 2012. Fluctuation in the functional activity of human colostrum phagocytes to Streptococcus pneumoniae and enteropathogenic Escherichia coli. J. Med. Microbiol. Diagn., 1: e104-e104. DOI: 10.4172/2161-0703.1000104

Franca, E.L., T.D.R. Nicomedes, I.D.M.P. Calderon and A.C.H. Franca, 2010. Time-dependent alterations of soluble and cellular components in human milk. Biol. Rhythm Res., 41: 333-347. DOI: 10.1080/09291010903407441

Franca, E.L., V.A. Silva, R.M.J. Volpato, P.A. Silva and M.F.S.S. Brune et al., 2013. Maternal Anemia induces changes in immunological and nutritional components of breast milk. J. Maternal Fetal Neonatal Med. DOI: 10.3109/14767058.2013.776529

Garofalo, R., 2010. Cytokines in human milk. J. Pediatr., 156: S36-40. DOI: 10.1016/j.jpeds.2009.11.019

Groen, M.W. and J.W. Beckstead, 2011. Multidimensional scaling of multiplex data: Human milk cytokines. Biol. Res. Nurs., 13: 289-296. DOI: 10.1177/1099800411402055

Hanson, L.A. and S.A. Silfverdal, 2009. The mother's immune system is a balanced threat to the foetus, turning to protection of the neonate. Acta Paediatr., 98: 221-228. DOI: 10.1111/j.1651-2227.2008.01143.x

Hara, C.D.C.P., A.C. Honorio-Franca, D.L.G. Fagundes, P.C. Guimaraes and E.L. Franca, 2013. Melatonin nanoparticles adsorbed to polyethylene glycol microspheres as activators of human colostrum macrophages. J. Nanomaterials, 2013: 973179973186. DOI: 10.1155/2013/973179

Haus, E. and M. Smolensky, 2006. Biological clocks and shift work: Circadian dysregulation and potential long-term effects. Cancer Causes Control, 17: 489500. DOI: $10.1007 / \mathrm{s} 10552-005-9015-4$ 
Haus, E., 2007. Chronobiology in the endocrine system. Adv. Drug Del. Rev., 59: 985-1014. DOI: 10.1016/j.addr.2007.01.001

Honorio-Franca, A.C., C.C.P. Hara, J.V.S. Ormonde, G.T. Nunes and E.L. Franca, 2013. Human colostrum melatonin exhibits a day-night variation and modulates the activity of colostral phagocytes. J. Appli. Biomed., 11: 153-162. DOI: 10.2478/v10136-012-0039-2

Kohut, M.L., A.E. Martin, D.S. Senchina and W. Lee, 2005. Glucocorticoids produced during exercise may be necessary for optimal virus-induced IL-2 and cell proliferation whereas both catecholamines and glucocorticoids may be required for adequate immune defense to viral infection. Brain Behav. Immunity, 19: 423-435. DOI: 10.1016/j.bbi.2005.04.006

Lack, L.C. and H.R.Wright, 2007. Chronobiology of sleep in humans. Cellular Mol. Life Sci., 64: 12051215. DOI: $10.1007 / \mathrm{s} 00018-007-6531-2$

Lim, H.Y., N. Muller, M.J. Herold, J.V.D. Brandt and H.M. Reichardt, 2007. Glucocorticoids exert opposing effects on macrophage function dependent on their concentration. Immunology, 122: 47-53. DOI: 10.1111/j.1365-2567.2007.02611.x

Markus, R.P. and Z.S. Ferreira, 2011. The immunepineal axis: The role of pineal and extra-pineal melatonin in modulating inflammation. Adv. Neuroimmune Biol., 1: 95-104. DOI: 10.3233/NIB2011-009

Morceli, G., A.C. Honorio-Franca, D.L.G. Fagundes, I.M.P. Calderon, E.L. Franca, 2013. Antioxidant effect of melatonin on the functional activity of colostral phagocytes in diabetic women. Plos One, 8: e56915-e56915. DOI: 10.1371/journal.pone.0056915
Morceli, G., E.L. Franca, V.B. Magalhaes, D.C. Damasceno and I.M.P. Calderon et al., 2011. Diabetes induced immunological and biochemical changes in human colostrum. Acta Paediatr., 100: 550-556. DOI: 10.1111/j.1651-2227.2010.02070.x

Prokesova, L., R. Lodinova-Zadnikova, J. Zizka, I. Kocourkova and O. Novotna et al., 2006. Cytokine levels in healthy and allergic mothers and their children during the first year of life. Pediatr. Allergy Immunol., 17: 75-183. DOI: 10.1111/j.13993038.2006.00395.x

Sauter, S.N., B. Roffler, C. Philipona, C. Morel and V. Rome et al., 2004. Intestinal development in neonatal calves: Effects of glucocorticoids and dependence on colostrum feeding. Biol. Neonate, 85: 94-104. DOI: 10.1159/000074965

Scheff, J.D., S.E. Calvano, S.F. Lowry and I.P. Androulakis, 2010. Modeling the influence of circadian rhythms on the acute inflammatory response. J. Theoret. Biol., 264: 1068-1076. DOI: 10.1016/j.jtbi.2010.03.026

Rivkees, S.A., 2003. Developing circadian rhythmicity in infants. Pediatrics, 112: 373-381. PMID: 12897290

Watson, C.J., C.H. Oliver and W.T. Khaled, 2011. Cytokine signalling in mammary gland development. J Reprod. Immunol., 88: 124-129. DOI: 10.1016/j.jri.2010.11.006

Xu, L., L. Zhang, Y. Zhang, Q. Sheng and A. Zhao, 2011. Qualitative and quantitative comparison of hormone contents between bovine and human colostrums. Int. Dairy J., 21: 54-57. DOI: 10.1016/j.idairyj.2010.04.006 